\title{
FIRST EIGENVALUE ESTIMATES OF DIRICHLET-TO-NEUMANN OPERATORS ON GRAPHS
}

\author{
BOBO HUA, YAN HUANG, AND ZUOQIN WANG
}

\begin{abstract}
Following Escobar Esc97 and Jammes Jam15, we introduce two types of isoperimetric constants and give lower bound estimates for the first nontrivial eigenvalues of Dirichlet-to-Neumann operators on finite graphs with boundary respectively.
\end{abstract}

\section{INTRODUCTION}

Let $(M, g)$ be a compact manifold with boundary $\partial M$. The Dirichlet-toNeumann operator $\Lambda: H^{\frac{1}{2}}(\partial M) \longrightarrow H^{-\frac{1}{2}}(\partial M)$ is defined as

$$
\Lambda(f)=\frac{\partial u_{f}}{\partial n},
$$

where $u_{f}$ is the harmonic extension of $f \in H^{\frac{1}{2}}(\partial M)$. The Dirichlet-toNeumann operator is a first order elliptic pseudo-differential operator Tay96, page 37] and its associated eigenvalue problem is also known as the Steklov problem, see $\mathrm{KKK}^{+} 14$ for a historical discussion. Since $\partial M$ is compact, the spectrum of $\Lambda$ is nonnegative, discrete and unbounded [Ban80, page95].

The Dirichlet-to-Neumann operator is closely related to the Calderón problem Cal80] of determining the anisotropic conductivity of a body from current and voltage measurements at its boundary. This makes it useful for applications to electrical impedance tomography, which is used in medical and geophysical imaging, see Uhl14 for a recent survey.

Eigenvalue estimates are of interest in spectral geometry. In Che70, Cheeger discovered a close relation between the geometric quantity, the isoperimetric constant (also called the Cheeger constant), and the analytic quantity, the first nontrivial eigenvalue of the Laplace-Beltrami operator on a closed manifold. Estimate of this type is called the Cheeger estimate.

For the first nontrivial eigenvalue of the Dirichlet-to-Neumann operator, two different types of lower bound estimates, which are similar to the classical Cheeger estimate, have been obtained by Escobar and Jammes respectively in Esc97] and Jam15. For convenience, we call them the Escobartype Cheeger estimate and the Jammes-type Cheeger estimate.

The Cheeger constant introduced by Escobar [Esc97, which we call the Escobar-type Cheeger constant, is defined as

B.H. is supported in part by NSFC, NO.11401106. Z.W is supported in part by NSFC, No. 11571131 and No. 11526212. 


$$
h_{E}(M):=\inf _{\operatorname{Area}(\Omega \cap \partial M) \leq \frac{1}{2} \operatorname{Area}(\partial M)} \frac{\operatorname{Area}(\partial \Omega \cap \operatorname{int}(M))}{\operatorname{Area}(\Omega \cap \partial M)},
$$

where Area $(\cdot)$ denotes the codimensional one Hausdorff measure, i.e. the Riemannian area, and $\operatorname{int}(M)$ denotes the interior of the manifold $M$. Let $\sigma_{1}$ be the first nontrivial eigenvalue of the Dirichlet-to-Neumann operator $\Lambda$, then the Escobar-type Cheeger estimate [Esc97, Theorem 10] reads as

$$
\sigma_{1} \geq \frac{\left(h_{E}(M) \mu_{1}(k)-a k\right) a}{a^{2}+\mu_{1}(k)},
$$

where $a>0, k>0$ are arbitrary positive constants, and $\mu_{1}(k)$ is the first eigenvalue of the following Robin problem

$$
\begin{cases}\Delta u+\mu_{1}(k) u=0, & \text { on } M, \\ \frac{\partial u}{\partial n}+k u=0, & \text { on } \partial M .\end{cases}
$$

The Jammes-type Cheeger constant, which was introduced in [Jam15], is defined as

$$
h_{J}(M)=\inf _{\operatorname{Vol}(\Omega) \leq \frac{1}{2} \operatorname{Vol}(M)} \frac{\operatorname{Area}(\partial \Omega \cap \operatorname{int}(M))}{\operatorname{Area}(\Omega \cap \partial M)},
$$

where $\operatorname{Vol}(\cdot)$ denotes the Riemannian volume. The Jammes-type Cheeger estimate [Jam15, Theorem 1] is given as follows

$$
\sigma_{1} \geq \frac{1}{4} h(M) h_{J}(M)
$$

where $h(M)$ is the classical Cheeger constant associated to the Laplacian operator with Neumann boundary condition on $M$, which is defined as

$$
h(M)=\inf _{\operatorname{Vol}(\Omega) \leq \frac{1}{2} \operatorname{Vol}(M)} \frac{\operatorname{Area}(\partial \Omega \cap \operatorname{int}(M))}{\operatorname{Vol}(\Omega)} .
$$

The Dirichlet-to-Neumann operator is naturally defined in the discrete setting. We recall some basic definitions on graphs. Let $V$ be a countable set which serves as the set of vertices of a graph and $\mu$ be a symmetric weight function given by

$$
\begin{aligned}
\mu: V \times V & \rightarrow[0, \infty), \\
(x, y) & \mapsto \mu_{x y}=\mu_{y x} .
\end{aligned}
$$

This induces a graph structure $G=(V, E)$ with the set of vertices $V$ and the set of edges $E$ which is defined as $\{x, y\} \in E$ if and only if $\mu_{x y}>0$, in symbols $x \sim y$. Note that we do allow self-loops in the graph, i.e. $x \sim x$ if $\mu_{x x}>0$. Given $\Omega_{1}, \Omega_{2} \subset V$, the set of edges between $\Omega_{1}$ and $\Omega_{2}$ is denoted by

$$
E\left(\Omega_{1}, \Omega_{2}\right):=\left\{e=\{x, y\} \in E \mid x \in \Omega_{1}, y \in \Omega_{2}\right\} .
$$

For any subset $\Omega \subset V$, there are two notions of boundary, i.e. the edge boundary and the vertex boundary. The edge boundary of $\Omega$, denoted by $\partial \Omega$, is defined as

$$
\partial \Omega:=E\left(\Omega, \Omega^{c}\right)
$$


where $\Omega^{c}:=V \backslash \Omega$. The vertex boundary of $\Omega$, denoted by $\delta \Omega$, is defined as $\delta \Omega:=\{x \in V \backslash \Omega \mid x \sim y$ for some $y \in \Omega\}$.

Set $\bar{\Omega}:=\Omega \cup \delta \Omega$. We introduce a measure on $\bar{\Omega}, m: \bar{\Omega} \rightarrow(0, \infty)$, as follows

$$
m_{x}= \begin{cases}\sum_{y \in V, y \sim x} \mu_{x y}, & x \in \Omega, \\ \sum_{y \in \Omega, y \sim x} \mu_{x y}, & x \in \delta \Omega .\end{cases}
$$

Accordingly, $m(A):=\sum_{x \in A} m_{x}$ denotes the measure of any subset $A \subset \bar{\Omega}$. Given any set $F$, we denote by $\mathbb{R}^{F}$ the collection of all real functions defined on $F$.

For any finite subset $\Omega \subset V$, in analogy to the Riemannian case, one can define the Dirichlet-to-Neumann operator in the discrete setting to be

$$
\begin{aligned}
\Lambda: \mathbb{R}^{\delta \Omega} & \rightarrow \mathbb{R}^{\delta \Omega}, \\
\varphi & \mapsto \Lambda \varphi:=\frac{\partial u_{\varphi}}{\partial n},
\end{aligned}
$$

where $u_{\varphi}$ is the harmonic extension of $\varphi$ to $\Omega$, and $\frac{\partial}{\partial n}$ is the outward normal derivative in the discrete setting defined as in (2.1) in section 2. We call $\Lambda$ the DtN operator for short. Let $\sigma(\Lambda)$ denote the spectrum of $\Lambda$. By the definition of $\Lambda$ and Green's formula, see Lemma 2.1, $\Lambda$ is a nonnegative self-adjoint operator, i.e. $\sigma(\Lambda)$ is a set of nonnegative real numbers. In fact, $\sigma(\Lambda)$ is contained in $[0,1]$, see Proposition 3.1.

It is well known that the eigenvalues of (normalized) Laplace operators on finite graphs without any boundary condition are contained in $[0,2]$. The multiplicity of eigenvalue 0 is equal to the number of connected components of the graph, see [BH12, Prop.1.3.7]. The largest possible eigenvalue 2 is achieved if and only if the graph is bipartite, see [Gri09, Theorem 2.3]. Similar results can be generalized to the case of DtN operators. For convenience, let $\widetilde{\Omega}$ denote the graph with vertices in $\bar{\Omega}$ and edges in $E(\Omega, \bar{\Omega})$, i.e.

$$
\widetilde{\Omega}:=(\bar{\Omega}, E(\Omega, \bar{\Omega})) .
$$

From Proposition 3.2, we know that the multiplicity of eigenvalue 0 of $\Lambda$ is equal to the number of connected components of $\widetilde{\Omega}$. Moreover, we show that the eigenspace associated to the eigenvalue 1 which is the largest possible eigenvalue is the kernel of a linear operator $Q$ whose definition is given in (3.2) below.

Given $A \subset \bar{\Omega}$, we denote by

$$
\partial_{\Omega} A:=\partial A \cap E(\Omega, \bar{\Omega})
$$

the relative boundary and $A^{\vee}:=\bar{\Omega} \backslash A$ the relative complement of $A$ in $\widetilde{\Omega}$. Without loss of generality, we always assume that $\widetilde{\Omega}$ is connected.

Now we consider the first nontrivial eigenvalue estimates of DtN operators on finite graphs. We introduce two isoperimetric constants following Escobar and Jammes, which we call Escobar-type Cheeger constant and Jammestype Cheeger constant respectively. 
Definition 1.1. The Escobar-type Cheeger constant for $\widetilde{\Omega}$ is defined as

$$
h_{E}(\widetilde{\Omega}):=\inf _{m(A \cap \delta \Omega) \leq \frac{1}{2} m(\delta \Omega)} \frac{\mu\left(\partial_{\Omega} A\right)}{m(A \cap \delta \Omega)} .
$$

The Jammes-type Cheeger constant for $\widetilde{\Omega}$ is defined as

$$
h_{J}(\widetilde{\Omega}):=\inf _{m(A) \leq \frac{1}{2} m(\bar{\Omega})} \frac{\mu\left(\partial_{\Omega} A\right)}{m(A \cap \delta \Omega)} .
$$

By definitions, one has $h_{J}(\widetilde{\Omega}) \leq h_{E}(\widetilde{\Omega})$, see Proposition 4.1. This estimate is optimal, see Example 4.1 in the paper.

To derive the Cheeger estimates, we first show that $h_{E}(\widetilde{\Omega})$ is equal to a type of Sobolev constant. Similar results can be found in both Riemannian and discrete case, see e.g. [Li12, Cha16, Chu97].

Theorem 1.1. Let $G$ be a finite graph and $\Omega \subset V$, then

$$
h_{E}(\widetilde{\Omega})=\inf _{f \in \mathbb{R}^{\bar{\Omega}}} \frac{\sum_{e=\{x, y\} \in E(\Omega, \bar{\Omega})} \mu_{x y}|f(x)-f(y)|}{\inf _{a \in \mathbb{R}} \sum_{x \in \delta \Omega} m_{x}|f(x)-a|} .
$$

Without loss of generality, we assume that the number of vertices in $\delta \Omega$ is at least two. From now on, we denote by $\lambda_{1}(\Omega)$ the first nontrivial eigenvalue of the DtN operator $\Lambda$ on $\Omega$ in a finite graph. In the discrete setting, it's easy to obtain an upper bound estimate as

$$
\lambda_{1}(\Omega) \leq 2 h_{E}(\widetilde{\Omega})
$$

see Proposition 4.2. The sharpness of this upper bound can be seen from Example 4.2. For the lower bound estimate, we obtain the Escobar-type Cheeger estimate as follows.

Theorem 1.2. Let $G$ be a finite graph and $\Omega \subset V$, then

$$
\lambda_{1}(\Omega) \geq \frac{\left(2 h_{E}(\widetilde{\Omega}) \mu_{1}(k)-a\left(k+\mu_{1}(k)\right)\right) a}{a^{2}+2 \mu_{1}(k)},
$$

where $a>0, k>0$ are arbitrary positive constants, and $\mu_{1}(k)$ is the first eigenvalue of the Robin problem

$$
\begin{cases}\Delta u+\mu_{1}(k) u=0, & \text { on } \Omega \\ \frac{\partial u}{\partial n}+k u=0, & \text { on } \partial \Omega .\end{cases}
$$

Analogous to the Riemannian case, we obtain Jammes-type Cheeger estimate following [Jam15].

Theorem 1.3. Let $G$ be a finite graph and $\Omega \subset V$, then

$$
\lambda_{1}(\Omega) \geq \frac{1}{2} h(\widetilde{\Omega}) h_{J}(\widetilde{\Omega}),
$$

where $h(\widetilde{\Omega})$ is the classical Cheeger constant of the graph $\widetilde{\Omega}$ viewed as a graph without any boundary condition. 
Remark 1.1. The Jammes-type Cheeger estimate is asymptotically sharp, see Example 5.1 .

For the completeness, we recall the definition of $h(\widetilde{\Omega})$,

$$
h(\widetilde{\Omega}):=\inf _{m(A) \leq \frac{1}{2} m(\bar{\Omega})} \frac{\mu\left(\partial_{\Omega} A\right)}{m(A)},
$$

where $A$ is any nonempty subset of $\bar{\Omega}$, see e.g. [Chu97, p.24]. By the definitions of $h(\widetilde{\Omega})$ and $h_{J}(\widetilde{\Omega})$, one is ready to see that $h(\widetilde{\Omega}) \leq h_{J}(\widetilde{\Omega})$. Notably, the classical Cheeger estimate uses only one Cheeger constant while the Jammes-type Cheeger estimate involves two. One may ask whether $\lambda_{1}(\Omega)$ can be bounded from below using only $h_{J}(\widetilde{\Omega})$. The answer is negative and we give a counterexample in Example 5.1.

As a corollary of Theorem 1.3 , we have the following interesting eigenvalue estimate, which has no counterpart in the Riemannian setting.

\section{Corollary 1.1.}

$$
\lambda_{1}(\Omega) \geq \frac{1}{8}\left(\zeta_{1}(\widetilde{\Omega})\right)^{2},
$$

where $\zeta_{1}(\widetilde{\Omega})$ is the first nontrivial eigenvalue of the Laplace operator with no boundary condition on $\widetilde{\Omega}$.

The organization of the paper is as follows: In Section 2, we collect some basic facts about the DtN operators on graphs. In Section 3, we study the spectrum of the DtN operators. In Section 4 and Section 5, we give the proof of the main theorems: Theorem 1.2 and 1.3 .

\section{PRELIMINARIES}

Let $(X, \nu)$ be a discrete measure space, i.e. $X$ is a discrete space equipped with a Borel measure $\nu$. The spaces of $\ell^{p}, p \in[1, \infty]$, summable functions on $(X, \nu)$, are defined routinely: Given a function $f \in \mathbb{R}^{X}$, for $p \in[1, \infty)$, we denote by

$$
\|f\|_{\ell^{p}}=\left(\sum_{x \in X}|f(x)|^{p} \nu(x)\right)^{1 / p},
$$

the $\ell^{p}$ norm of $f$. For $p=\infty$,

$$
\|f\|_{\ell^{\infty}}=\sup _{x \in X}|f(x)| .
$$

Let $\ell^{p}(X, \nu):=\left\{f \in \mathbb{R}^{X} \mid\|f\|_{\ell^{p}}<\infty\right\}$ be the space of $\ell^{p}$ summable functions on $(X, \nu)$. In our setting, these definitions apply to $(X, \nu)=(\Omega, m)$ or $(\delta \Omega, m)$. The case where $p=2$ is of particular interest, as we have the Hilbert spaces $\ell^{2}(\Omega, m):=\left\{f \in \mathbb{R}^{\Omega} \mid\|f\|_{\ell^{2}}<\infty\right\}$ and $\ell^{2}(\delta \Omega, m):=\{\varphi \in$ 
$\left.\mathbb{R}^{\delta \Omega} \mid\|\varphi\|_{\ell^{2}}<\infty\right\}$ equipped with the standard inner products

$$
\begin{aligned}
\langle f, g\rangle_{\Omega} & =\sum_{x \in \Omega} f(x) g(x) m_{x}, \quad f, g: \Omega \rightarrow \mathbb{R}, \\
\langle\varphi, \psi\rangle_{\delta \Omega} & =\sum_{x \in \delta \Omega} \varphi(x) \psi(x) m_{x}, \quad \varphi, \psi: \delta \Omega \rightarrow \mathbb{R} .
\end{aligned}
$$

Given $\Omega \subset V$, an associated quadratic form is defined as

$$
D_{\Omega}(f, g)=\sum_{e=\{x, y\} \in E(\Omega, \bar{\Omega})} \mu_{x y}(f(x)-f(y))(g(x)-g(y)), \quad f, g \in \mathbb{R}^{\bar{\Omega}} .
$$

The Dirichlet energy of $f \in \mathbb{R}^{\bar{\Omega}}$ can be written as

$$
D_{\Omega}(f):=D_{\Omega}(f, f) \text {. }
$$

For any $f \in \mathbb{R}^{\bar{\Omega}}$, the Laplacian operator is defined as

$$
\Delta f(x):=\frac{1}{m_{x}} \sum_{y \in V: y \sim x} \mu_{x y}(f(y)-f(x)), \quad x \in \Omega
$$

and the outward normal derivative of $f$ is defined as

$$
\frac{\partial f}{\partial n}(z):=\frac{1}{m_{z}} \sum_{x \in \Omega: x \sim z} \mu_{z x}(f(z)-f(x)), \quad z \in \delta \Omega .
$$

We recall the following two well-known results on Laplace operators.

Lemma 2.1. (Green's formula) Let $f, g \in \mathbb{R}^{\bar{\Omega}}$. Then

$$
\langle\Delta f, g\rangle_{\Omega}=-D_{\Omega}(f, g)+\left\langle\frac{\partial f}{\partial n}, g\right\rangle_{\delta \Omega} .
$$

Lemma 2.2. Given any $\varphi \in \mathbb{R}^{\delta \Omega}$, there is a unique function $u_{\varphi} \in \mathbb{R}^{\bar{\Omega}}$ satisfying the Laplace equation

$$
\Delta u_{\varphi}(x)=0, \quad x \in \Omega,
$$

and the boundary condition

$$
u_{\varphi}(x)=\varphi(x), \quad x \in \delta \Omega .
$$

Remark 2.1. We will denote by $u_{\varphi}$ the harmonic extension of $\varphi \in \mathbb{R}^{\delta \Omega}$ to $\bar{\Omega}$ in this paper. For the proof of Lemma 2.1 and 2.2, one can see e.g. [Gri09].

Proposition 2.1. $\Lambda$ is a nonnegative self-adjoint linear operator on $\ell^{2}(\delta \Omega, m)$.

Proof. For any $f, g \in \ell^{2}(\delta \Omega)$, by Green's formula, we have

Hence

$$
\begin{aligned}
& 0=\left\langle\Delta u_{f}, u_{g}\right\rangle_{\Omega}=-D_{\Omega}\left(u_{f}, u_{g}\right)+\left\langle\frac{\partial f}{\partial n}, g\right\rangle_{\delta \Omega}, \\
& 0=\left\langle u_{f}, \Delta u_{g}\right\rangle_{\Omega}=-D_{\Omega}\left(u_{f}, u_{g}\right)+\left\langle f, \frac{\partial g}{\partial n}\right\rangle_{\delta \Omega} .
\end{aligned}
$$

$$
\langle\Lambda f, g\rangle=\langle f, \Lambda g\rangle
$$


In particular,

$$
0=\left\langle\Delta u_{f}, u_{f}\right\rangle_{\Omega}=-D_{\Omega}\left(u_{f}, u_{f}\right)+\left\langle\frac{\partial f}{\partial n}, f\right\rangle_{\delta \Omega},
$$

then

$$
\langle\Lambda f, f\rangle_{\delta \Omega}=D_{\Omega}\left(u_{f}, u_{f}\right) \geq 0 .
$$

So we complete the proof.

For any $y \in \delta \Omega$, let $\delta_{y}(z)$ denote the delta function at $y$, i.e. $\delta_{y}(y)=1$ and $\delta_{y}(z)=0$ for any $z \in \delta \Omega, z \neq y$. We denote by $P(\cdot, y)$ the solution of equation (2.3) with Dirichlet boundary condition $\frac{1}{m_{y}} \delta_{y}$. The family $\{P(\cdot, y)\}_{y \in \delta \Omega}$ are called Poission kernels associated to the Dirchlet boundary conditions on $\Omega$. They have interesting probabilistic explanations using simple random walks, see e.g. [Law10, p.25] and [LL10, chapter 8]. Using Poission kernels, one can regard the DtN operator on $\Omega$ as a Laplace operator defined on a graph with the set of vertices $\delta \Omega$ and modified edges.

Proposition 2.2. The DtN operator can be written as

$$
\Lambda \varphi(x)=\frac{1}{m_{x}} \sum_{y \in \delta \Omega} \widetilde{\mu}_{x y}(\varphi(x)-\varphi(y))
$$

where $\widetilde{\mu}_{x y}=\sum_{z \in \Omega} \mu_{x z} P(z, y) m_{y}$ and $\sum_{y \in \delta \Omega} \widetilde{\mu}_{x y}=m_{x}$.

Proof. For any given $\varphi \in \mathbb{R}^{\delta \Omega}$, we have

$$
\varphi(x)=\sum_{y \in \delta \Omega} \varphi(y) m_{y} \cdot \frac{\delta_{y}(x)}{m_{y}} .
$$

By the linearity of equation 2.3), we have

$$
u_{\varphi}(x)=\sum_{y \in \delta \Omega} \varphi(y) m_{y} P(x, y) .
$$

Hence by the definition of $\Lambda$,

$$
\begin{aligned}
\Lambda \varphi(x) & =\frac{1}{m_{x}} \sum_{z \in \Omega} \mu_{x z}\left(\varphi(x)-\sum_{y \in \delta \Omega} \varphi(y) m_{y} P(z, y)\right) \\
& =\varphi(x)-\frac{1}{m_{x}} \sum_{y \in \delta \Omega}\left(\sum_{z \in \Omega} \mu_{x z} P(z, y) m_{y}\right) \varphi(y) \\
& =\varphi(x)-\frac{1}{m_{x}} \sum_{y \in \delta \Omega} \widetilde{\mu}_{x y} \varphi(y) .
\end{aligned}
$$


Notice that $u_{\varphi}(x) \equiv 1$, if we choose $\varphi(x)=1, x \in \delta \Omega$. Hence combining with (2.4), we have

$$
\begin{aligned}
\sum_{y \in \delta \Omega} \widetilde{\mu}_{x y} & =\sum_{z \in \Omega} \mu_{x z}\left(\sum_{y \in \delta_{e} \Omega} P(z, y) m_{y}\right) \\
& =\sum_{z \in \Omega} \mu_{x z} \cdot 1=m_{x} .
\end{aligned}
$$

Then the proposition follows.

From Proposition 2.2, the DtN operator $\Lambda$ can be written in a matrix form. We define matrices $D_{\delta \Omega}, A_{\delta \Omega \times \Omega}, P_{\Omega \times \delta \Omega}$ as

$$
\begin{aligned}
\left(D_{\delta \Omega}\right)_{x y} & =m_{x} \delta_{x}(y), & & x, y \in \delta \Omega, \\
\left(A_{\delta \Omega \times \Omega}\right)_{x z} & =\mu_{x z}, & & x \in \delta \Omega, z \in \Omega, \\
\left(P_{\Omega \times \delta \Omega}\right)_{z x} & =P(z, x), & & x \in \delta \Omega, z \in \Omega .
\end{aligned}
$$

Then we have

Corollary 2.1. The DtN operator $\Lambda$ can be written as

$$
\Lambda=I-D^{-1} A P D
$$

where $I$ is the identity map.

\section{Spectrum of the DtN operator}

Given $\varphi \in \mathbb{R}^{\delta \Omega}$, for simplicity we denote by $\bar{\varphi}$ the null-extension of $\varphi$ to $\bar{\Omega}$, which is defined as

$$
\bar{\varphi}(x)= \begin{cases}0, & x \in \Omega \\ \varphi(x), & x \in \delta \Omega\end{cases}
$$

For any $p \in[1, \infty]$, the $\ell^{p}-\ell^{p}$ norm of the operator $\Lambda$ is defined as

$$
\|\Lambda\|_{p, p}:=\sup _{\varphi \in \mathbb{R}^{\delta \Omega},\|\varphi\|_{\ell^{p}=1}}\|\Lambda \varphi\|_{\ell^{p}}
$$

Proposition 3.1. The $\ell^{2}-\ell^{2}$ and $\ell^{\infty}-\ell^{\infty}$ norm of the operator $\Lambda$ are bounded, in particular

$$
\|\Lambda\|_{2,2} \leq 1, \quad\|\Lambda\|_{\infty, \infty} \leq 2
$$


Proof. Let $\varphi \in \mathbb{R}^{\delta \Omega}$. According to Hölder's inequality,

$$
\begin{aligned}
\|\Lambda \varphi\|_{\ell^{2}(\delta \Omega, m)}^{2} & =\sum_{x \in \delta \Omega} m_{x}\left|\sum_{y \in \Omega} \frac{\mu_{x y}}{m_{x}}\left(\varphi(x)-u_{\varphi}(y)\right)\right|^{2} \\
& \leq \sum_{x \in \delta \Omega} \sum_{y \in \Omega} \mu_{x y}\left|\varphi(x)-u_{\varphi}(y)\right|^{2} \leq D_{\Omega}\left(u_{\varphi}\right) \\
& \leq D_{\Omega}(\bar{\varphi})=\sum_{x \in \delta \Omega, y \in \Omega} \mu_{x y} \varphi^{2}(x) \\
& =\|\varphi\|_{\ell^{2}(\delta \Omega, m)}^{2},
\end{aligned}
$$

where (3.1) follows from the fact that harmonic functions minimize the Dirichlet energy in the class of functions with the same boundary condition. This proves the $\ell^{2}-\ell^{2}$ norm bound.

For the $\ell^{\infty}-\ell^{\infty}$ norm bound, we have

$$
\begin{aligned}
\|\Lambda \varphi\|_{\infty} & =\sup _{x \in \delta \Omega}\left|\frac{1}{m_{x}} \sum_{y \in \Omega} \mu_{x y}\left(\varphi(x)-u_{\varphi}(y)\right)\right| \\
& \leq \sup _{x \in \delta \Omega} \frac{1}{m_{x}} \sum_{y \in \Omega} \mu_{x y}\left|\left(\varphi(x)-u_{\varphi}(y)\right)\right| \\
& \leq 2\|\varphi\|_{\infty} .
\end{aligned}
$$

Remark 3.1. From Proposition 3.1, we have $\sigma(\Lambda) \subset[0,1]$.

By interpolation, we have

Corollary 3.1. $\|\Lambda\|_{p, p} \leq 2^{1-\theta}$, where $\theta \in[0,1)$ and $p=\frac{2}{\theta} \in[2, \infty]$.

Proposition 3.2. The multiplicity of the eigenvalue 0 of $\Lambda$, i.e. $\operatorname{dim} \operatorname{Ker} \Lambda$, is equal to the number of connected components of the graph $\widetilde{\Omega}$.

Proof. Let $\varphi \in \mathbb{R}^{\delta \Omega}$ be an eigenfunction associated to eigenvalue 0 of $\Lambda$, i.e. $\Lambda \varphi=0$. By Green's formula we have

$$
0=\left\langle\Delta u_{\varphi}, u_{\varphi}\right\rangle_{\Omega}=-D_{\Omega}\left(u_{\varphi}\right)+\langle\Lambda \varphi, \varphi\rangle_{\delta \Omega}=-D_{\Omega}\left(u_{\varphi}\right)
$$

Hence $u_{\varphi}$ is constant on each connected component of $\widetilde{\Omega}$.

From now on, we always assume that the graph $\widetilde{\Omega}$ is connected and $\Lambda$ is an operator from $\ell^{2}(\delta \Omega, m)$ to $\ell^{2}(\delta \Omega, m)$. Let

$$
E_{1}(\Lambda):=\left\{\varphi \in \mathbb{R}^{\delta \Omega} \mid \Lambda \varphi=\varphi\right\}
$$

be the space of eigenvectors associated to the eigenvalue 1 which might be empty. Set $\delta_{I} \Omega:=\{x \in \Omega \mid x \sim y$ for some $y \in \delta \Omega\}$. For any $\varphi \in \mathbb{R}^{\delta \Omega}$, we 
introduce a linear operator $Q: \mathbb{R}^{\delta \Omega} \rightarrow \mathbb{R}^{\delta_{I} \Omega}$, which is defined as

$$
Q \varphi(x)=\frac{1}{m_{x}} \sum_{y \in \delta \Omega} \mu_{x y} \varphi(y), \quad x \in \delta_{I} \Omega .
$$

Let $\sharp \delta \Omega\left(\sharp \delta_{I} \Omega\right.$ resp.) denote the number of vertices in $\delta \Omega\left(\delta_{I} \Omega\right.$ resp. $)$. We order the eigenvalues of the DtN operator $\Lambda$ in the nondecreasing way:

$$
0=\lambda_{0}(\Omega)<\lambda_{1}(\Omega) \leq \cdots \leq \lambda_{N-1}(\Omega) \leq 1,
$$

where $N=\sharp \delta \Omega$.

We obtain some characterisations of $E_{1}(\Lambda)$ in the following proposition.

Proposition 3.3. (1) For $\varphi \in \mathbb{R}^{\delta \Omega}, \varphi \in E_{1}(\Lambda)$ if and only if

$$
u_{\varphi}=\bar{\varphi}
$$

$$
E_{1}(\Lambda)=\operatorname{Ker} Q
$$

Proof. (1) For the "if " part,

$$
\Lambda \varphi(x)=\frac{1}{m_{x}} \sum_{y \in \Omega} \mu_{x y}(\bar{\varphi}(x)-\bar{\varphi}(y))=\varphi(x) .
$$

For the "only if" part, if $\varphi \in E_{1}(\Lambda)$, then all the inequalities in (3.1) are equalities. Hence $D_{\Omega}\left(u_{\varphi}\right)=D_{\Omega}(\bar{\varphi})$. This implies that $\bar{\varphi}$ attains the minimal Dirichlet energy with fixed boundary condition, i.e. $\bar{\varphi}$ is harmonic. By the uniqueness of harmonic functions with fixed boundary condition, we have $\bar{\varphi}=u_{\varphi}$.

(2) If $\varphi \in E_{1}(\Lambda)$, then $u_{\varphi}=\bar{\varphi}$. For any $x \in \delta_{I} \Omega$,

$$
\begin{aligned}
Q \varphi(x) & =\frac{1}{m_{x}} \sum_{y \in \delta \Omega} \mu_{x y} \varphi(y) \\
& =\frac{1}{m_{x}}\left(\sum_{y \in \delta \Omega} \mu_{x y}(\bar{\varphi}(y)-\bar{\varphi}(x))+\sum_{y \in \Omega} \mu_{x y}(\bar{\varphi}(y)-\bar{\varphi}(x))\right) \\
& =\Delta u_{\varphi}(x)=0 .
\end{aligned}
$$

Hence $E_{1}(\Lambda) \subset \operatorname{Ker} Q$. On the other hand, If $\varphi \in \operatorname{Ker} Q$, then for any $x \in \delta_{I} \Omega$,

$$
\Delta \bar{\varphi}(x)=\frac{1}{m_{x}} \sum_{y \in \delta \Omega} \mu_{x y}(\bar{\varphi}(y)-\bar{\varphi}(x))=\frac{1}{m_{x}} \sum_{y \in \delta \Omega} \mu_{x y} \varphi(y)=0 .
$$

Hence $u_{\varphi}=\bar{\varphi}$, i.e. $\operatorname{Ker} Q \subset E_{1}(\Lambda)$, and the proof is completed.

Remark 3.2. By Proposition 3.3, the problem of determining $E_{1}(\Lambda)$ can be reduced to the properties of the combinatorial structure of $\delta_{I} \Omega \cup \delta \Omega$, which is independent of the inner structure $\Omega \backslash \delta_{I} \Omega$. 
From Proposition 3.3 , we obtain a sufficient condition for $E_{1}(\Lambda)$ to be nonempty as follows.

\section{Corollary 3.2.}

$$
\operatorname{dim} E_{1}(\Lambda) \geq \sharp \delta \Omega-\sharp \delta_{I} \Omega .
$$

In particular, $E_{1}(\Lambda) \neq \emptyset$ if $\sharp \delta \Omega>\sharp \delta_{I} \Omega$.

Proof. It directly follows from the fact that $\operatorname{dim} \operatorname{Ker} Q+\operatorname{dim} \operatorname{Im} Q=\sharp \delta \Omega$.

\section{4. escobar-type Cheeger estimate}

Proposition 4.1. Let $G$ be a finite graph and $\Omega \subset V$, then we have

$$
h_{J}(\widetilde{\Omega}) \leq h_{E}(\widetilde{\Omega}) .
$$

Proof. Choose $A \subset \bar{\Omega}$ that achieves $h_{E}(\widetilde{\Omega})$, i.e.

$$
m(A \cap \delta \Omega) \leq \frac{m(\delta \Omega)}{2} \quad \text { and } \quad h_{E}(\widetilde{\Omega})=\frac{\mu\left(\partial_{\Omega} A\right)}{m(A \cap \delta \Omega)} .
$$

If $m(A) \leq \frac{m(\bar{\Omega})}{2}$, then $h_{J}(\widetilde{\Omega}) \leq h_{E}(\widetilde{\Omega})$. If $m(A) \geq \frac{m(\bar{\Omega})}{2}$, i.e. $m\left(A^{c}\right) \leq$ $\frac{m(\bar{\Omega})}{2}$, then

$$
\frac{\mu\left(\partial_{\Omega} A^{c}\right)}{m\left(A^{c} \cap \delta \Omega\right)}=\frac{\mu\left(\partial_{\Omega} A\right)}{m\left(A^{c} \cap \delta \Omega\right)} \leq \frac{\mu\left(\partial_{\Omega} A\right)}{m(A \cap \delta \Omega)} .
$$

Hence in both cases we have $h_{J}(\widetilde{\Omega}) \leq h_{E}(\widetilde{\Omega})$.

From the following example, we know that the equality in the above proposition can be achieved.

Example 4.1. Consider the path graph $P_{6}$ as shown in Figure 1 with unit edge weights, $\Omega=\left\{v_{2}, v_{3}, v_{4}, v_{5}\right\}$ and $\delta \Omega:=\left\{v_{1}, v_{6}\right\}$. By computation, we have $h_{E}(\widetilde{\Omega})=h_{J}(\widetilde{\Omega})=1$.

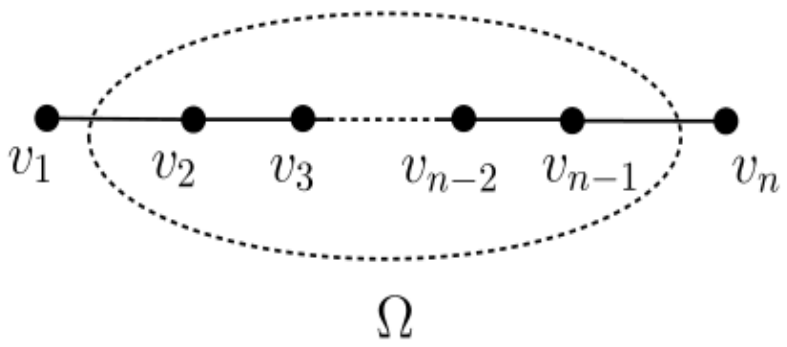

FiguRe 1.

For convenience, we need the following notion. 
Definition 4.1. For any $f \in \mathbb{R}^{\delta \Omega}$, we call $k \in \mathbb{R}$ the $L^{1}$-mean value of $f$ over $\delta \Omega$ if $k$ satisfies

$$
m(\{x \in \delta \Omega \mid f(x) \geq k\}) \geq \frac{1}{2} m(\delta \Omega)
$$

and

$$
m(\{x \in \delta \Omega \mid f(x) \leq k\}) \geq \frac{1}{2} m(\delta \Omega) .
$$

Remark 4.1. The $L^{1}$-mean value may not be unique in general. For simplicity, we denote by $\bar{f}$ the $L^{1}$-mean value of $f \in \mathbb{R}^{\delta \Omega}$.

\section{Lemma 4.1.}

$$
\underset{k \in \mathbb{R}}{\arg \min } \sum_{x \in \delta \Omega} m_{x}|f(x)-k|=\bar{f}
$$

where $\arg \min$ denotes the value $k$ at which $\sum_{x \in \delta \Omega} m_{x}|f(x)-k|$ attains the minimum.

Remark 4.2. (a) For the proof of Lemma 4.1, one refers to e.g. [CSZ15].

(b) From Lemma 4.1. Theorem 1.1 is equivalent to

$$
h_{E}(\widetilde{\Omega})=\inf _{f \in \mathbb{R}_{\bar{\Omega}}} \frac{\sum_{e=\{x, y\} \in E(\Omega, \bar{\Omega})} \mu_{x y}|f(x)-f(y)|}{\sum_{x \in \delta \Omega} m_{x}|f(x)-\bar{f}|} .
$$

We will need the following discrete version of Co-area formula. For discrete Co-area formula, see e.g. Gri09, Lemma 3.3].

Lemma 4.2. For any $f \in \mathbb{R}^{\bar{\Omega}}$, we have

$$
\sum_{e=\{x, y\} \in E(\Omega, \bar{\Omega})} \mu_{x y}|f(x)-f(y)|=\int_{-\infty}^{\infty} \sum_{e=\{x, y\} \in E(\Omega, \bar{\Omega}), f(x)<\sigma \leq f(y)} \mu_{x y} d \sigma .
$$

Proof. For any interval $(a, b]$, we denote by $\chi_{(a, b]}$ the characteristic function on $(a, b]$, i.e.

$$
\begin{aligned}
\chi_{(a, b]}(x)=\left\{\begin{array}{ll}
0, & x \notin(a, b], \\
1, & x \in(a, b] .
\end{array} \sum_{-\infty} \mu_{x y} d \sigma\right. & =\int_{-\infty}^{\infty} \sum_{e=\{x, y\} \in E(\Omega, \bar{\Omega})} \mu_{x y} \chi_{(f(x), f(y)]}(\sigma) d \sigma \\
& =\sum_{e=\{x, y\} \in E(\Omega, \bar{\Omega})} \mu_{x y} \int_{-\infty}^{\infty} \chi_{(f(x), f(y)]}(\sigma) d \sigma \\
& =\sum_{e=\{x, y\} \in E(\Omega, \bar{\Omega})} \mu_{x y}|f(x)-f(y)| .
\end{aligned}
$$

Now we are ready to prove Theorem 1.1 . 
Proof of Theorem 1.1. From Remark 4.2, it suffices to prove (4.1). Choose $A \subset \bar{\Omega}$ that achieves $h_{E}(\widetilde{\Omega})$, i.e.

$$
m(A \cap \delta \Omega) \leq m\left(A^{\vee} \cap \delta \Omega\right), \quad h_{E}(\widetilde{\Omega})=\frac{\mu\left(\partial_{\Omega} A\right)}{m(A \cap \delta \Omega)} .
$$

Set

$$
u(x)= \begin{cases}1, & x \in A \\ 0, & x \in \bar{\Omega} \backslash A .\end{cases}
$$

We observe that $\bar{u}$, the $L^{1}$-mean value of $u$, is contained in $[0,1]$. To see this, one just need to notice that if $m(A \cap \delta \Omega)=m\left(A^{\vee} \cap \delta \Omega\right)$, then $\bar{u} \in[0,1]$; if $m(A \cap \delta \Omega)<m\left(A^{\vee} \cap \delta \Omega\right)$, then $\bar{u}=0$.

For any $t \in[0,1]$,

$$
\begin{aligned}
\sum_{x \in \delta \Omega} m_{x}|u(x)-t| & =\sum_{x \in A \cap \delta \Omega} m_{x}(1-t)+\sum_{x \in A^{\vee} \cap \delta \Omega} m_{x} t \\
& =m(A \cap \delta \Omega)+t \cdot\left(m\left(A^{\vee} \cap \delta \Omega\right)-m(A \cap \delta \Omega)\right) \\
& \geq m(A \cap \delta \Omega) .
\end{aligned}
$$

Hence

$$
\frac{\sum_{e=\{x, y\} \in E(\Omega, \bar{\Omega})} \mu_{x y}|u(x)-u(y)|}{\sum_{x \in \delta \Omega} m_{x}|u(x)-\bar{u}|} \leq \frac{\mu(\partial A \cap E(\Omega, \bar{\Omega}))}{m(A \cap \delta \Omega)}=h_{E}(\widetilde{\Omega}) .
$$

Then it follows that

$$
h_{E}(\widetilde{\Omega}) \geq \inf _{f \in \mathbb{R}^{\bar{\Omega}}} \frac{\sum_{e=\{x, y\} \in E(\Omega, \bar{\Omega})} \mu_{x y}|f(x)-f(y)|}{\sum_{x \in \delta \Omega} m_{x}|f(x)-\bar{f}|} .
$$

Now we prove the opposite direction. For any nonconstant function $f \in \mathbb{R}^{\bar{\Omega}}$, choose a constant $c$ such that

$$
\begin{aligned}
& m(\{f<c\} \cap \delta \Omega) \leq m(\{f \geq c\} \cap \delta \Omega), \\
& m(\{f \leq c\} \cap \delta \Omega) \geq m(\{f>c\} \cap \delta \Omega) .
\end{aligned}
$$

Set $g:=f-c$, then we have

$$
m(\{g<\sigma\} \cap \delta \Omega) \leq m(\{g \geq \sigma\} \cap \delta \Omega), \quad \text { for } \quad \sigma \leq 0
$$

and

$$
m(\{g<\sigma\} \cap \delta \Omega) \geq m(\{g \geq \sigma\} \cap \delta \Omega), \quad \text { for } \quad \sigma>0 .
$$

For any $\sigma \in \mathbb{R}$, set

$$
G(\sigma):=\sum_{e=\{x, y\} \in E(\Omega, \bar{\Omega}), g(x)<\sigma \leq g(y)} \mu_{x y} .
$$

Then by Lemma 4.2, we have

$$
\sum_{e=\{x, y\} \in E(\Omega, \bar{\Omega})} \mu_{x y}|f(x)-f(y)|=\int_{-\infty}^{\infty} G(\sigma) d \sigma .
$$


Set $A:=\{g<\sigma\}$ for $\sigma \leq 0$ and $A:=\{g \geq \sigma\}$ for $\sigma>0$. In either case, we have $m(A \cap \delta \Omega) \leq m\left(A^{\vee} \cap \delta \Omega\right)$ and

$$
G(\sigma) \geq h_{E}(\widetilde{\Omega}) \cdot m(A \cap \delta \Omega)=h_{E}(\widetilde{\Omega}) \cdot \begin{cases}m(\{g<\sigma\} \cap \Omega), & \text { for } \quad \sigma \leq 0 \\ m(\{g \geq \sigma\} \cap \Omega), & \text { for } \sigma>0\end{cases}
$$

by the definition of $h_{E}(\widetilde{\Omega})$. Hence

$$
\begin{aligned}
& \sum_{e=\{x, y\} \in E(\Omega, \bar{\Omega})} \mu_{x y}|f(x)-f(y)|=\int_{-\infty}^{0} G(\sigma) d \sigma+\int_{0}^{\infty} G(\sigma) d \sigma \\
\geq & h_{E}(\widetilde{\Omega})\left(\int_{-\infty}^{0} m(\{g<\sigma\} \cap \Omega) d \sigma+\int_{0}^{\infty} m(\{g \geq \sigma\} \cap \Omega) d \sigma\right) \\
= & h_{E}(\widetilde{\Omega})\left(\int_{-\infty}^{0} m(\{g<\sigma\} \cap \Omega) d \sigma+\int_{0}^{\infty} m(\{g \geq \sigma\} \cap \Omega) d \sigma\right) \\
= & h_{E}(\widetilde{\Omega})\left(\int_{-\infty}^{0} \sum_{x \in \delta \Omega} \chi_{(g(x), 0]} m_{x} d \sigma+\int_{0}^{\infty} \sum_{x \in \delta \Omega} \chi_{(0, g(x)]} m_{x} d \sigma\right) \\
= & h_{E}(\widetilde{\Omega}) \sum_{x \in \delta \Omega}|f(x)-c| m_{x} .
\end{aligned}
$$

Then the other direction follows and we complete the proof of the theorem.

We obtain the following upper bound estimate for $\lambda_{1}(\Omega)$.

Proposition 4.2. Let $G$ be a finite graph and $\Omega \subset V$, then we have

$$
\lambda_{1}(\Omega) \leq 2 h_{E}(\widetilde{\Omega}) .
$$

Proof. Choose $A \subset \bar{\Omega}$ that achieves $h_{E}(\widetilde{\Omega})$, i.e. $m(A \cap \delta \Omega) \leq \frac{1}{2} m(\delta \Omega)$ and $\frac{\mu\left(\partial_{\Omega} A\right)}{m(A \cap \delta \Omega)}=h_{E}(\widetilde{\Omega})$. Set $\varphi(x) \in \mathbb{R}^{\delta \Omega}$ as

$$
\varphi(x)= \begin{cases}\frac{1}{m(A \cap \delta \Omega)}, & x \in A \cap \delta \Omega, \\ -\frac{1}{m\left(A^{\vee} \cap \delta \Omega\right)}, & x \in A^{\vee} \cap \delta \Omega .\end{cases}
$$

Similarly set $\widetilde{\varphi}(x)$ to be

$$
\widetilde{\varphi}(x)= \begin{cases}\frac{1}{m(A \cap \delta \Omega)}, & x \in A, \\ -\frac{1}{m\left(A^{\vee} \cap \delta \Omega\right)}, & x \in A^{\vee} .\end{cases}
$$

Then we have

$$
\begin{aligned}
\lambda_{1}(\Omega) & \leq \frac{D_{\Omega}\left(u_{\varphi}\right)}{\sum_{x \in \delta \Omega} \varphi^{2}(x) m_{x}} \leq \frac{D_{\Omega}(\widetilde{\varphi})}{\sum_{x \in \delta \Omega} \varphi^{2}(x) m_{x}} \\
& =\left(\frac{1}{m(A \cap \delta \Omega)}+\frac{1}{m\left(A^{\vee} \cap \delta \Omega\right)}\right) \mu(\partial A \cap E(\Omega, \bar{\Omega})) \\
& \leq 2 \frac{\mu\left(\partial_{\Omega} A\right)}{m(A \cap \delta \Omega)}=2 h_{E}(\widetilde{\Omega}) .
\end{aligned}
$$


The second inequality follows from the fact that harmonic functions minimize the Dirichlet energy among functions with the same boundary condition.

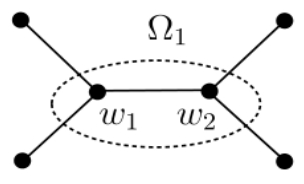

$G_{1}$

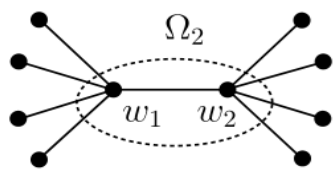

$G_{2}$

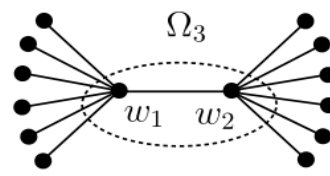

$G_{3}$

FiguRe 2.

Remark 4.3. The above upper bound estimate for $\lambda_{1}(\Omega)$ is sharp. From the following example, we can see that the factor 2 in the upper bound estimate can't be reduced.

Example 4.2. Consider a sequence of graphs $\left\{G_{n}\right\}_{n=1}^{\infty}$ as shown in Figure 2 with $\Omega_{n}=\left\{w_{1}, w_{2}\right\}, \delta \Omega_{n}=\left\{v_{1}, v_{2}, \cdots, v_{4 n}\right\}$ and unit edge weights. By calculation, $\lambda_{1}\left(G_{n}\right)=\frac{1}{n+1}$ and $h_{E}\left(G_{n}\right)=\frac{1}{2 n}$. Hence $\lambda_{1}\left(G_{n}\right)=\frac{1}{n+1} \leq \frac{1}{n}=$ $2 h_{E}\left(G_{n}\right)$.

At the end of this section, we give the proof of Theorem 1.2 .

Proof of Theorem 1.2. Let $u$ be the first eigenvector associated to $\lambda_{1}(\Omega)$. For simplicity, we still denote by $u$ the harmonic extension of $u$. Set $v=$ $(u-\bar{u})_{+}$and choose $\bar{v}=0$. Then

$$
m(\{v=0\} \cap \delta \Omega) \geq \frac{1}{2} m(\delta \Omega) .
$$

Applying Theorem 1.1 by choosing $f=v^{2}$, we have

$$
h_{E}(\widetilde{\Omega}) \cdot \sum_{x \in \delta \Omega} v^{2}(x) m_{x} \leq \sum_{e=\{x, y\} \in E(\Omega, \bar{\Omega})} \mu_{x y}\left|v^{2}(x)-v^{2}(y)\right| .
$$

For the right hand side of the above inequality,

$$
\begin{aligned}
& \left(\sum_{e=\{x, y\} \in E(\Omega, \bar{\Omega})} \mu_{x y}\left|v^{2}(x)-v^{2}(y)\right|\right)^{2} \\
\leq & \sum_{e=\{x, y\} \in E(\Omega, \bar{\Omega})} \mu_{x y}(v(x)+v(y))^{2} \cdot \sum_{e=\{x, y\} \in E(\Omega, \bar{\Omega})} \mu_{x y}(v(x)-v(y))^{2} \\
\leq & 2 \sum_{e=\{x, y\} \in E(\Omega, \bar{\Omega})} \mu_{x y}\left(v^{2}(x)+v^{2}(y)\right) \cdot \sum_{e=\{x, y\} \in E(\Omega, \bar{\Omega})} \mu_{x y}(v(x)-v(y))^{2} .
\end{aligned}
$$


Notice that it suffices to consider the graph $\widetilde{\Omega}$, then

$$
\begin{aligned}
& \sum_{e=\{x, y\} \in E(\Omega, \bar{\Omega})} \mu_{x y}\left(v^{2}(x)+v^{2}(y)\right) \\
= & \frac{1}{2} \sum_{x, y \in \Omega} \mu_{x y}\left(v^{2}(x)+v^{2}(y)\right)+\sum_{x \in \Omega} \sum_{y \in \delta \Omega} \mu_{x y}\left(v^{2}(x)+v^{2}(y)\right) \\
= & \sum_{x \in \Omega} v^{2}(x)\left(\sum_{y \in \Omega}+\sum_{y \in \delta \Omega}\right) \mu_{x y}+\sum_{y \in \delta \Omega} v^{2}(y) \sum_{x \in \Omega} \mu_{x y} \\
= & \sum_{x \in \bar{\Omega}} v^{2}(x) m_{x} .
\end{aligned}
$$

Hence

$$
h_{E}(\widetilde{\Omega}) \cdot \sum_{x \in \delta \Omega} v^{2}(x) m_{x} \leq\left(2 \sum_{x \in \bar{\Omega}} v^{2}(x) m_{x} \cdot \sum_{e=\{x, y\} \in E(\Omega, \bar{\Omega})} \mu_{x y}(v(x)-v(y))^{2}\right)^{1 / 2},
$$

i.e.

$$
h_{E}(\widetilde{\Omega}) \cdot \sum_{x \in \delta \Omega}(u-\bar{u})_{+}^{2} m_{x} \leq\left(2 \sum_{x \in \bar{\Omega}}(u-\bar{u})_{+}^{2} m_{x} \cdot \sum_{e=\{x, y\} \in E(\Omega, \bar{\Omega})} \mu_{x y}(u(x)-u(y))^{2}\right)^{1 / 2} .
$$

Similarly, we have

$$
h_{E}(\widetilde{\Omega}) \cdot \sum_{x \in \delta \Omega}(u-\bar{u})_{-}^{2} m_{x} \leq\left(2 \sum_{x \in \bar{\Omega}}(u-\bar{u})_{-}^{2} m_{x} \cdot \sum_{e=\{x, y\} \in E(\Omega, \bar{\Omega})} \mu_{x y}(u(x)-u(y))^{2}\right)^{1 / 2} .
$$

Hence

$$
\begin{aligned}
& h_{E}(\widetilde{\Omega}) \cdot \sum_{x \in \delta \Omega}(u-\bar{u})^{2} m_{x} \\
\leq & \left(2 \sum_{x \in \bar{\Omega}}(u-\bar{u})^{2} m_{x} \cdot \sum_{e=\{x, y\} \in E(\Omega, \bar{\Omega})} \mu_{x y}(u(x)-u(y))^{2}\right)^{1 / 2} \\
\leq & \frac{a}{2} \sum_{x \in \bar{\Omega}}(u-\bar{u})^{2} m_{x}+\frac{1}{a} \sum_{e=\{x, y\} \in E(\Omega, \bar{\Omega})} \mu_{x y}(u(x)-u(y))^{2} \\
= & \frac{a}{2}\left(\sum_{x \in \Omega}+\sum_{x \in \delta \Omega}\right)(u-\bar{u})^{2} m_{x}+\frac{1}{a} \sum_{e=\{x, y\} \in E(\Omega, \bar{\Omega})} \mu_{x y}(u(x)-u(y))^{2} .
\end{aligned}
$$

For any $\varphi \in \mathbb{R}^{\bar{\Omega}}$ we have

$$
\mu_{1}(k) \leq \frac{D_{\Omega}(\varphi)+k \sum_{x \in \delta \Omega} \varphi^{2}(x) m_{x}}{\sum_{x \in \Omega} \varphi^{2}(x) m_{x}} .
$$


Hence

$$
\sum_{x \in \Omega}(u-\bar{u})^{2} m_{x} \leq \frac{D_{\Omega}(u)}{\mu_{1}(k)}+\frac{k}{\mu_{1}(k)} \sum_{x \in \delta \Omega}(u-\bar{u})^{2} m_{x} .
$$

Combining with the above inequality and 4.2 , we have

$$
\left(h_{E}(\widetilde{\Omega})-\frac{a\left(k+\mu_{1}(k)\right)}{2 \mu_{1}(k)}\right) \frac{2 a \mu_{1}(k)}{a^{2}+2 \mu_{1}(k)} \leq \frac{D_{\Omega}(u)}{\sum_{x \in \delta \Omega}(u-\bar{u})^{2} m_{x}} .
$$

Using the fact that $u$ is the first eigenfunction associated to $\lambda_{1}$ we find that

$$
\left(h_{E}(\widetilde{\Omega})-\frac{a\left(k+\mu_{1}(k)\right)}{2 \mu_{1}(k)}\right) \frac{2 a \mu_{1}(k)}{a^{2}+2 \mu_{1}(k)} \leq \frac{\lambda_{1} \sum_{x \in \delta \Omega} u^{2}(x) m_{x}}{\sum_{x \in \delta \Omega}(u-\bar{u})^{2} m_{x}} .
$$

Since $\langle u, 1\rangle_{\delta \Omega}=0$, we have

$$
\frac{\sum_{x \in \delta \Omega} u^{2}(x) m_{x}}{\sum_{x \in \delta \Omega}(u-\bar{u})^{2} m_{x}} \leq 1
$$

and therefore

$$
\begin{aligned}
\lambda_{1}(\Omega) & \geq\left(h_{E}(\widetilde{\Omega})-\frac{a\left(k+\mu_{1}(k)\right)}{2 \mu_{1}(k)}\right) \frac{2 a \mu_{1}(k)}{a^{2}+2 \mu_{1}(k)} \\
& =\frac{\left(2 h_{E}(\widetilde{\Omega}) \mu_{1}(k)-a\left(k+\mu_{1}(k)\right)\right) a}{a^{2}+2 \mu_{1}(k)} .
\end{aligned}
$$

Remark 4.4. The maximum of the right hand side of $(1.2)$ with respect to $a$ can be achieved at

$$
a_{0}=\frac{2 h \mu}{\sqrt{(k+\mu)^{2}+2 h^{2} \mu}+(k+\mu)}
$$

and the maximum is

$$
\begin{gathered}
\frac{h^{2} \mu \sqrt{(k+\mu)^{2}+2 h^{2} \mu}}{2 h^{2} \mu+(k+\mu)^{2}+(k+\mu) \sqrt{(k+\mu)^{2}+2 h^{2} \mu}} . \\
\text { 5. Jammes-Type CheEger Estimate }
\end{gathered}
$$

Proposition 5.1. Let $G$ be a finite graph and $\Omega \subset V$, then we have

$$
h_{J}(\widetilde{\Omega}) \leq 1 \text {. }
$$

Proof. Choose $A=\{v\}$, where $v \in \delta \Omega$. Then by the definition of $h_{J}(\widetilde{\Omega})$, we have $h_{J}(\widetilde{\Omega}) \leq \frac{\mu\left(\partial_{\Omega} A\right)}{m(A \cap \delta \Omega)}=\frac{m_{v}}{m_{v}}=1$.

The eigenvalues of $\Lambda$ can be characterised by Rayleigh quotient as follows

$$
\begin{gathered}
\lambda_{1}(\Omega)=\inf _{\varphi \in \mathbb{R}^{\delta \Omega},\|\varphi\|_{\ell^{2}}=1, \varphi \perp 1}\langle\Lambda \varphi, \varphi\rangle=\inf _{\varphi \in \mathbb{R}^{\delta \Omega,},\|\varphi\|_{\ell^{2}}=1, \varphi \perp 1} D_{\Omega}\left(u_{\varphi}\right), \\
\lambda_{N-1}(\Omega)=\sup _{\varphi \in \mathbb{R}^{\delta \Omega},\|\varphi\|_{\ell^{2}}=1}\langle\Lambda \varphi, \varphi\rangle=\sup _{\varphi \in \mathbb{R}^{\delta \Omega,\|\varphi\|_{\ell^{2}}=1}} D_{\Omega}\left(u_{\varphi}\right) .
\end{gathered}
$$


We denote by $\sigma_{1}$ the first nontrivial eigenvalue of the Dirichlet-to-Neumann operator on a compact manifold with boundary. For convenience, we recall the idea of the proof of Jammes-type Cheeger estimate, which can be divided into four steps.

Step 1: Choosing $f$ as the eigenfunction associated to $\sigma_{1}$, we still denote by $f$ the harmonic extension of $f$ to $M$ with $\operatorname{vol}\left(M^{+}\right) \leq \frac{\operatorname{vol}(M)}{2}$, where $M^{+}:=\{x \in M \mid f(x)>0\}$.

Step 2: Show that $\sigma_{1}=\frac{\int_{M^{+}}|d f|^{2}}{\int_{\partial M^{+}} f^{2}}$, where $\partial M^{+}=M^{+} \cap \partial M$.

Step 3: By Hölder's inequality, $\sigma_{1}=\frac{\left(\int_{M^{+}} f^{2}\right)\left(\int_{M^{+}}|d f|^{2}\right)}{\left(\int_{M^{+}} f^{2}\right)\left(\int_{\partial M^{+}} f^{2}\right)} \geq \frac{1}{4} \frac{\left(\int_{M^{+}}\left|d\left(f^{2}\right)\right|\right)^{2}}{\left(\int_{M^{+}} f^{2}\right)\left(\int_{\partial M^{+}} f^{2}\right)}$.

Step 4: Set $D_{t}:=f^{-1}([\sqrt{t}, \infty)), \partial_{I} D_{t}:=\partial D_{t} \cap \operatorname{int}(M)$ and $\partial_{E} D_{t}:=\partial D_{t} \backslash$ $\partial_{I} D_{t}$. Use Co-area formula to show that $\int_{M^{+}}\left|d\left(f^{2}\right)\right|=\int_{t \geq 0} \operatorname{Area}\left(\partial_{I} D_{t}\right)$, $\int_{M^{+}} f^{2}=\int_{t>0} \operatorname{vol}\left(D_{t}\right)$ and $\int_{\partial M^{+}} f^{2}=\int_{t>0} \operatorname{Area}\left(\partial_{E} D_{t}\right)$.

Then by the definitions of $h_{M}$ and $h_{J}(M)$ the lower bound estimate of $\sigma_{1}$ follows.

Inspired by the Riemannian case, we can prove the Jammes-type Cheeger constant for $\lambda_{1}(\Omega)$ in the discrete setting. Let $0 \neq f \in \mathbb{R}^{\delta \Omega}$ be an eigenfunction associated to $\lambda_{1}(\Omega)$. For convenience, we still denote $u_{f}$ by $f(x)$. Set $\bar{\Omega}^{+}=\{x \in \bar{\Omega} \mid f(x)>0\}$ with $m\left(\bar{\Omega}^{+}\right) \leq \frac{m(\bar{\Omega})}{2}$ (otherwise we can change the sign of $f$ ) and set

$$
g(x)= \begin{cases}f(x), & x \in \bar{\Omega}^{+}, \\ 0, & \text { otherwise. }\end{cases}
$$

For simplicity, we set $\bar{\Omega}^{-}:=\bar{\Omega} \backslash \bar{\Omega}^{+}, \Omega^{+}:=\bar{\Omega}^{+} \cap \Omega, \Omega^{-}:=\Omega \backslash \Omega^{+}, \delta^{+} \Omega:=$ $\bar{\Omega}^{+} \cap \delta \Omega$ and $\delta^{-} \Omega:=\delta \Omega \backslash \delta^{+} \Omega$. In order to prove Jammes-type Cheeger estimate, we need the following Lemmas.

Lemma 5.1. For $g$ as in (5.1), we have

$$
\lambda_{1}(\Omega) \geq \frac{\sum_{e=\{x, y\} \in E\left(\bar{\Omega}^{+}, \bar{\Omega}\right)} \mu_{x y}(g(y)-g(x))^{2}}{\sum_{x \in \delta^{+} \Omega} g^{2}(x) m_{x}} .
$$

Proof. Notice that it suffices to consider graph $\widetilde{\Omega}$. Hence for any $x \in \delta \Omega$, we have

$$
\Delta f(x)=\frac{1}{m_{x}} \sum_{y \in \Omega} \mu_{x y}(f(y)-f(x))=-\frac{\partial f(x)}{\partial n} .
$$

Then

$$
\begin{aligned}
\langle\Delta f(x), g(x)\rangle_{\bar{\Omega}^{+}} & =\sum_{x \in \Omega^{+}} \Delta f(x) g(x) m_{x}+\sum_{x \in \delta^{+} \Omega} \Delta f(x) g(x) m_{x} \\
& =-\lambda_{1}(\Omega) \sum_{x \in \delta^{+} \Omega} g^{2}(x) m_{x} .
\end{aligned}
$$


Notice that

$$
\begin{aligned}
& \langle\Delta f(x), g(x)\rangle_{\bar{\Omega}^{+}}=\left(\sum_{x \in \bar{\Omega}^{+}} \sum_{y \in \bar{\Omega}^{+}}+\sum_{x \in \bar{\Omega}^{+}} \sum_{y \in \bar{\Omega}^{-}}\right) \mu_{x y}(f(y)-f(x)) g(x) \\
= & -\frac{1}{2} \sum_{x, y \in \bar{\Omega}^{+}} \mu_{x y}(f(y)-f(x))(g(y)-g(x))-\sum_{x \in \bar{\Omega}^{+}} \sum_{y \in \bar{\Omega}^{-}} \mu_{x y}(f(x)-f(y))(g(x)-g(y)) \\
\leq & -\sum_{e=\{x, y\} \in E\left(\bar{\Omega}^{+}, \bar{\Omega}^{+}\right)} \mu_{x y}(g(y)-g(x))^{2}-\sum_{x \in \bar{\Omega}^{+}} \sum_{y \in \bar{\Omega}^{-}} \mu_{x y}(g(x)-g(y))^{2} \\
= & -\sum_{e=\{x, y\} \in E\left(\bar{\Omega}^{+}, \bar{\Omega}\right)} \mu_{x y}(g(y)-g(x))^{2} .
\end{aligned}
$$

Then the lemma follows in view of (5.3).

Multiplying both the numerator and denominator of the fraction in the right hand side of $(5.2)$ by $\sum_{x \in \bar{\Omega}^{+}} g^{2}(x) m_{x}$ and setting

$$
\frac{P}{Q}:=\frac{\sum_{x \in \bar{\Omega}^{+}} g^{2}(x) m_{x} \cdot \sum_{e=\{x, y\} \in E\left(\bar{\Omega}^{+}, \bar{\Omega}\right)} \mu_{x y}(g(y)-g(x))^{2}}{\sum_{x \in \bar{\Omega}^{+}} g^{2}(x) m_{x} \cdot \sum_{x \in \delta^{+} \Omega} g^{2}(x) m_{x}},
$$

we have

$$
\lambda_{1}(\Omega) \geq \frac{P}{Q}
$$

\section{Lemma 5.2.}

$$
P \geq \frac{1}{2}\left(\sum_{e=\{x, y\} \in E\left(\bar{\Omega}^{+}, \bar{\Omega}\right)}\left|g^{2}(x)-g^{2}(y)\right| \mu_{x y}\right)^{2} .
$$

Proof. Note that

$$
\begin{aligned}
& \sum_{x \in \bar{\Omega}^{+}} g^{2}(x) m_{x}=\left(\sum_{x \in \bar{\Omega}^{+}} \sum_{y \in \bar{\Omega}^{+}}+\sum_{x \in \bar{\Omega}^{+}} \sum_{y \in \bar{\Omega}^{-}}\right) g^{2}(x) \mu_{x y} \\
= & \frac{1}{2} \sum_{x, y \in \bar{\Omega}^{+}}\left(g^{2}(x)+g^{2}(y)\right) \mu_{x y}+\sum_{x \in \bar{\Omega}^{+}} \sum_{y \in \bar{\Omega}^{-}} g^{2}(x) \mu_{x y} \\
= & \sum_{e=\{x, y\} \in E\left(\bar{\Omega}^{+}, \bar{\Omega}^{+}\right)}\left(g^{2}(x)+g^{2}(y)\right) \mu_{x y}+\sum_{x \in \bar{\Omega}^{+}} \sum_{y \in \bar{\Omega}^{-}} g^{2}(x) \mu_{x y} \\
= & \sum_{e=\{x, y\} \in E\left(\bar{\Omega}^{+}, \bar{\Omega}\right)}\left(g^{2}(x)+g^{2}(y)\right) \mu_{x y} .
\end{aligned}
$$


Hence

$$
\begin{aligned}
P & =\sum_{e=\{x, y\} \in E\left(\bar{\Omega}^{+}, \bar{\Omega}\right)}\left(g^{2}(x)+g^{2}(y)\right) \mu_{x y} \cdot \sum_{e=\{x, y\} \in E\left(\bar{\Omega}^{+}, \bar{\Omega}\right)} \mu_{x y}(g(y)-g(x))^{2} \\
& \geq \frac{1}{2} \sum_{e=\{x, y\} \in E\left(\bar{\Omega}^{+}, \bar{\Omega}\right)}(g(x)+g(y))^{2} \mu_{x y} \cdot \sum_{e=\{x, y\} \in E\left(\bar{\Omega}^{+}, \bar{\Omega}\right)} \mu_{x y}(g(y)-g(x))^{2} \\
& \geq \frac{1}{2}\left(\sum_{e=\{x, y\} \in E\left(\bar{\Omega}^{+}, \bar{\Omega}\right)}\left|g^{2}(x)-g^{2}(y)\right| \mu_{x y}\right)^{2} .
\end{aligned}
$$

The last inequality follows from Hölder's inequality.

For any $t>0$, set $D_{t}:=g^{-1}([\sqrt{t},+\infty))=\left\{x \in \bar{\Omega} \mid g^{2}(x) \geq t\right\}$. Then we have $m\left(D_{t}\right) \leq m\left(\bar{\Omega}^{+}\right) \leq \frac{m(\bar{\Omega})}{2}$.

\section{Lemma 5.3.}

$$
\int_{0}^{\infty} \mu\left(\partial D_{t} \cap E(\Omega, \bar{\Omega})\right) d t=\sum_{e=\{x, y\} \in E\left(\bar{\Omega}^{+}, \bar{\Omega}\right)} \mu_{x y}\left|g^{2}(x)-g^{2}(y)\right| .
$$

Proof. It follows from Lemma 4.2 by setting $f=g^{2}$ and considering the edge set $E\left(\bar{\Omega}^{+}, \bar{\Omega}\right)$.

\section{Lemma 5.4.}

$$
\begin{gathered}
\int_{0}^{\infty} m\left(D_{t}\right) d t=\sum_{x \in \bar{\Omega}^{+}} g^{2}(x) m_{x} . \\
\int_{0}^{\infty} m\left(D_{t} \cap \delta \Omega\right) d t=\sum_{x \in \delta^{+} \Omega} g^{2}(x) m_{x} .
\end{gathered}
$$

Proof. Similar to Lemma 4.2, we have

$$
\int_{0}^{\infty} m\left(D_{t}\right) d t=\int_{0}^{\infty} \sum_{x \in D_{t}} m_{x} d t=\int_{0}^{\infty} \sum_{x \in \bar{\Omega}^{+}} m_{x} \chi_{\left(0, g^{2}(x)\right]}(t) d t=\sum_{x \in \bar{\Omega}^{+}} g^{2}(x) m_{x}
$$

and

$$
\int_{0}^{\infty} m\left(D_{t} \cap \delta \Omega\right) d t=\int_{0}^{\infty} \sum_{x \in \delta^{+} \Omega} m_{x} \chi_{\left(0, g^{2}(x)\right]}(t) d t=\sum_{x \in \delta^{+} \Omega} g^{2}(x) m_{x} .
$$

Now we are ready to prove Theorem 1.3 . 
Proof of Theorem 1.3. Combining with the above Lemmas (Lemma 5.1 to 5.4), we have

$$
\begin{aligned}
\lambda_{1}(\Omega) & \geq \frac{1}{2} \frac{\int_{0}^{\infty} \mu\left(\partial D_{t} \cap E(\Omega, \bar{\Omega})\right) d t \cdot \int_{0}^{\infty} \mu\left(\partial D_{t} \cap E(\Omega, \bar{\Omega})\right) d t}{\sum_{x \in \bar{\Omega}^{+}} g^{2}(x) m_{x} \cdot \sum_{x \in \delta^{+} \Omega} g^{2}(x) m_{x}} \\
& \geq \frac{1}{2} \frac{\int_{0}^{\infty} h(\widetilde{\Omega}) m\left(D_{t}\right) d t \cdot \int_{0}^{\infty} h_{J}(\widetilde{\Omega}) m\left(D_{t} \cap \delta \Omega\right) d t}{\sum_{x \in \bar{\Omega}^{+}} g^{2}(x) m_{x} \cdot \sum_{x \in \delta^{+} \Omega} g^{2}(x) m_{x}} \\
& =\frac{h(\widetilde{\Omega}) h_{J}(\widetilde{\Omega})}{2} \frac{\int_{0}^{\infty} m\left(D_{t}\right) d t \cdot \int_{0}^{\infty} m\left(D_{t} \cap \delta \Omega\right) d t}{\sum_{x \in \bar{\Omega}^{+}} g^{2}(x) m_{x} \cdot \sum_{x \in \delta^{+} \Omega} g^{2}(x) m_{x}} \\
& =\frac{h(\widetilde{\Omega}) h_{J}(\widetilde{\Omega})}{2} .
\end{aligned}
$$

From Theorem 1.3 , we know that $h(\widetilde{\Omega})$ plays an important role in Jammestype Cheeger estimate. Recall that $\zeta_{1}(\widetilde{\Omega})$ is the first nontrivial eigenvalue of the Laplace operator with no boundary condition on $\widetilde{\Omega}$ and the classical Cheeger estimates reads as

$$
2 h(\widetilde{\Omega}) \geq \zeta_{1}(\widetilde{\Omega}) \geq \frac{h(\widetilde{\Omega})^{2}}{2},
$$

see [Chu97, p.26]. Then we are ready to prove Corollary 1.1

Proof of Corollary 1.1. Recall that $h(\widetilde{\Omega}) \leq h_{J}(\widetilde{\Omega})$. Combining with Theorem 1.3 and (5.5), we have

$$
\lambda_{1}(\Omega) \geq \frac{h(\widetilde{\Omega})^{2}}{2} \geq \frac{\left(\zeta_{1}(\widetilde{\Omega})\right)^{2}}{8} .
$$

Finally we give an example to show that $\lambda_{1}$ can't be bounded from below using only $h_{J}(\widetilde{\Omega})$.

Example 5.1. Consider a path graph with even vertices $n(n \geq 6)$ and unit edge weights as shown in Figure 1. Set $\Omega=\left\{v_{2}, v_{3}, \cdots, v_{n-1}\right\}, \delta_{E} \Omega=$ $\left\{v_{1}, v_{n}\right\}$. Then we have

$$
\Lambda=\left(\begin{array}{cc}
\frac{1}{n-1} & -\frac{1}{n-1} \\
-\frac{1}{n-1} & \frac{1}{n-1}
\end{array}\right) .
$$

Hence $\lambda_{0}(\Omega)=0$ and $\lambda_{1}(\Omega)=\frac{2}{n-1}$. Choosing $A=\left\{v_{1}, v_{2}, \cdots, v_{\frac{n}{2}}\right\}$, we obtain that $h(\widetilde{\Omega})=\frac{1}{n-1}$ and $h_{J}(\widetilde{\Omega})=1$. Hence the Jammes-type Cheeger estimate we obtained is asymptotically sharp of the same order $\frac{1}{n-1}$ on both sides as $n \rightarrow \infty$. Moreover, one can not obtain that $\lambda_{1}(\Omega) \geq F\left(h_{J}(\widetilde{\Omega})\right)$ for any positive function $F$. 


\section{REFERENCES}

[Ban80] C. Bandle. Isoperimetric inequalities and applications, volume 7 of Monographs and Studies in Mathematics. Pitman (Advanced Publishing Program), Boston, Mass.-London, 1980.

[BH12] A.E. Brouwer and W.H. Haemers. Spectra of graphs. Universitext. Springer, New York, 2012.

[Cal80] A.P. Calderón. On an inverse boundary value problem. Seminar on Numerical Analysis and its Applications to Continuum Physics, Rio de Janeiro, Editors W.H. Meyer and M.A. Raupp, Sociedade Brasileira de Matematica, pages 6573, 1980.

[Cha16] K. C. Chang. Spectrum of the 1-Laplacian and Cheeger's constant on graphs. J. Graph Theory, 81(2):167-207, 2016.

[Che70] J. Cheeger. A lower bound for the smallest eigenvalue of the Laplacian. In Problems in analysis (Papers dedicated to Salomon Bochner, 1969), pages 195199. Princeton Univ. Press, Princeton, NJ, 1970.

[Chu97] F.R.K. Chung. Spectral Graph Theory, volume 92 of CBMS Regional Conference Series in Mathematics. Published for the Conference Board of the Mathematical Sciences, Washington, DC; by the American Mathematical Society, Providence, RI, 1997.

[CSZ15] K. C. Chang, Sihong Shao, and Dong Zhang. The 1-Laplacian Cheeger cut: theory and algorithms. J. Comput. Math., 33(5):443-467, 2015.

[Esc97] J. Escobar. The geometry of the first non-zero Stekloff eigenvalue. Journal of Functional Analysis, 150(2):544-556, 1997.

[Gri09] A. Grigor'yan. Analysis on graphs. Lecture Notes, University of Bielefeld, 2009. https://www.math.uni-bielefeld.de/ grigor/aglect.pdf

[Jam15] P. Jammes. Une inégalité de Cheeger pour le spectre de Steklov. Annales de l'Institut Fourier, 65(3):1381-1385, 2015.

$\left[\mathrm{KKK}^{+} 14\right]$ N. Kuznetsov, T. Kulczycki, M. Kwasnicki, A. Nazarov, B. Siudeja, S. Poborchi, and I. Polterovich. The legacy of Vladimir Andreevich Steklov. Notices of the American Mathematical Society, 61(1):pgs. 9-23, 2014.

[Law10] Gregory F. Lawler. Random walk and the heat equation, volume 55 of Student Mathematical Library. American Mathematical Society, Providence, RI, 2010.

[Li12] P. Li. Geometric analysis, volume 134 of Cambridge Studies in Advanced Mathematics. Cambridge University Press, Cambridge, 2012.

[LL10] Gregory F. Lawler and V. Limic. Random walk: a modern introduction, volume 123 of Cambridge Studies in Advanced Mathematics. Cambridge University Press, Cambridge, 2010.

[Tay96] M.E. Taylor. Partial differential equations. II. Qualitative studies of linear equations, volume 116 of Applied Mathematical Sciences. Springer-Verlag, New York, 1996.

[Uhl14] G. Uhlmann. Inverse problems: seeing the unseen. Bull. Math. Sci., 4(2):209279, 2014. 
School of Mathematical Sciences, Lmns, Fudan University, Shanghai 200433, China; Shanghai Center for Mathematical Sciences, Fudan University, ShangHAI 200433, CHINA

E-mail address: bobohua@fudan.edu.cn

School of Mathematical Sciences, Fudan University, Shanghai 200433, China.

E-mail address: yanhuang0509@gmail.com

School of Mathematical Sciences, University of Science and Technology of China, Hefei, Anhui 230026, China.

E-mail address: wangzuoq@ustc.edu.cn 